\title{
DETC2013-12361
}

\section{VIBRATION BASED SUN GEAR DAMAGE DETECTION}

\author{
Adrian Hood* \\ Kelsen LaBerge \\ U.S. Army Research Laboratory \\ Aberdeen Proving Ground, MD
}

\author{
David Lewicki \\ NASA Glenn Research Center \\ Cleveland, $\mathrm{OH}$
}

\author{
Darryll Pines \\ A. James Clark School of Engineering \\ University of Maryland, College Park, MD
}

\begin{abstract}
Seeded fault experiments were conducted on the planetary stage of an $\mathrm{OH}-58 \mathrm{C}$ helicopter transmission. Two vibration based methods are discussed that isolate the dynamics of the sun gear from that of the planet gears, bearings, input spiral bevel stage, and other components in and around the gearbox. Three damaged sun gears: two spalled and one cracked, serve as the focus of this current work. A non-sequential vibration separation algorithm was developed and the resulting signals analyzed. The second method uses only the time synchronously averaged data but takes advantage of the signal/source mapping required for vibration separation. Both algorithms were successful in identifying the spall damage. Sun gear damage was confirmed by the presence of sun mesh groups. The sun tooth crack condition was inconclusive.
\end{abstract}

\section{NOMENCLATURE}

$N_{\text {Extract }} \quad$ Number of extractions

$N_{i j}^{*}, N_{i j}^{* *} \quad$ Extraction \& Mapping Index

$N_{H T} \quad$ Hunting Tooth Ratio

$P_{i}, A_{j} \quad$ Planet $i$, Accelerometer $j$

$\ell_{E}, \ell_{v}, N_{T P}$ Number of points: extraction,VSW,TMP

$N_{\text {pin }}, N_{\text {bev }}$ Number of teeth: spiral bevel pinion and gear.

$N_{s}, N_{p}, N_{r}$ Number of teeth: sun, ring, planet and ring respectively

$\theta_{s}, \theta_{p}, \theta_{c}$ Rotation angles: sun, planet, carrier respectively

$\overline{\theta_{S}^{1}}, \theta_{A}^{j}, \theta_{P}^{i} \quad$ Angles: Initial ST1, $A_{j}, P_{i}$

\footnotetext{
${ }^{*}$ This material is declared a work of the U.S. Government and is not subject to copyright protection in the United States. Approved for public release; distribution is unlimited. Email: adrian.a.hood@us.army.mil
}

$\begin{array}{ll}\theta_{\text {sep }}^{i j}, \theta_{s 1}^{i j} & \text { Separation angle between } P_{i} \text { and } A_{j} \text {, Local angle of ST1 } \\ \Delta \theta_{r, p, s} & \text { Tooth Pitch: Ring, Planet, Sun } \\ M_{v} & \text { Number of TMPs contained in each extraction } \\ \bar{N} & \text { Number of interpolated points per carrier cycle } \\ N_{\text {avg }} & \text { Avg. number of points per carrier cycle (pre-interpolation) } \\ P_{i}^{k} & \text { kth Tooth ID on } P_{i} \\ S T n & \text { Sun Tooth } n \\ P_{b} & \text { Gear Pitch } \\ \psi_{i} & \text { Planet spacing } \\ T M P & \text { Tooth Mesh Period } \\ \text { VSW } & \text { Vibration Separation Waveform } \\ \text { HTA } & \text { Hunting Tooth Average } \\ \overline{H T n} & \text { HTA of HT Group } n \\ \text { SGVS } & \text { Sun Gear Vibration Separation } \\ \text { PGVS } & \text { Planet Gear Vibration Separation } \\ \text { SASP } & \text { Single Accelerometer / Single Planet } \\ \text { GSMM } & \text { Geometrically Synchronized Measurement Method }\end{array}$

\section{INTRODUCTION}

The U.S. Army desires to use Condition Based Maintenance $(\mathrm{CBM}+)$ for its fleet of vehicles [1]. The premise is that critical components are serviced when indicators reveal that they can no longer function as designed. In Army rotorcraft, the high drive system maintenance cost and failure consequences justify the need over traditional schedule based maintenance.

Significant research has been devoted to health and usage monitoring systems (HUMS) for drive systems as researchers have attempted to develop qualitative and quantitative helicopter gear fault detection methods [2-19]. Typical research methods 
reference a current observation to a previous measurement taken at a known state (often undamaged) while employing signal processing and statistical algorithms to detect deviations that correlate to damage. A false positive, or false alarm, will unnecessarily remove a rotorcraft from service requiring costly inspection and maintenance. While false positives result in loss of time and money, false negatives have more drastic repercussions, leading to accidents and loss of life. Thus, the number of false positives and negatives must be kept to a minimum in order for $\mathrm{CBM}+$ to be cost effective and reliable. The pathway for improving probability of correct diagnosis involves understanding the gear system's fundamental behavior. In this paper, we will study the relationship between mechanical response and damage as well as damage and sensor response using seeded fault testing.

Planetary transmissions are used on many rotorcraft in the final stage of the main-rotor drive systems as they provide an efficient, compact method to reduce speed. They, however, exhibit unique challenges relating to gear and bearing fault detection. First, multiple planets are contained in such systems. Since these planets all operate at the same speeds and loads, healthy components could potentially mask the vibration signatures of faulty components. Second, the planet gears exhibit epicyclic motion about a sun gear. Thus, the location of a faulty component, such as a planet tooth defect, changes position in time going through mesh in different locations relative to the gearbox housing. This could provide a challenge for a fixed-position accelerometer (usually mounted on the transmission housing) used to monitor component health. There has been some recent work in the development of planetary fault detection, but most of these efforts were not validated for helicopter applications [20-28].

This work combines two separate research programs to detect planetary faults. One, a collaboration between NASA, U. S. Army Research Laboratory (ARL) and the University of Maryland (UMD), investigated damaged sun gears, a tri-plex bearing spall, and planet gear bearing faults on both the OH-58A and $\mathrm{C}$ models. Data was collected using an array of accelerometers, microphones, torsional accelerometers, and an oil debris sensor. Another was a collaboration between ARL, NASA, the Army's Aviation Applied Technology Directorate (AATD), and Bell Helicopter under the Operations Support and Sustainment Technologies (OSST) program. This work investigated 15 faults associated with the planet gears, sun gears, ring gears, and planet bearing using the same OH-58C transmission [29]. Data was collected using accelerometers, fiber optic strain gauges, and oil debris sensors.

The goal of this paper is to present the results of two vibration based techniques used to detect seeded faults in sun gears.

\section{VIBRATION SEPARATION}

Gears mesh with a characteristic vibration signature which changes as the system becomes damaged. Analysis of plane- tary gear dynamics is complicated by the simultaneous meshing of multiple teeth. For the transmission under study, the frequency spectrum is rich with components due to the pinion/bevel gear mesh of the first stage and the sun/planet/ring gear mesh of the second stage. Also included are components arising from surrounding structures, such as the end gear mesh. Time synchronous averaging has proven to be an effective tool to isolate non-commensurate dynamics in transmissions. While this technique may be able to isolate the planetary system from others, it has shown to be ineffective in isolating the individual components of the planetary system [30].

In 1990, McFadden introduced a method called vibration separation that allows for standard vibration based condition indicators to be applied directly to individual components of a planetary transmission [21]. It is a signal processing technique used on planetary gear systems for the purpose of isolating dynamics associated with a single planet, sun, or ring gear. McFadden's technique focused on isolating planets but was later modified to handle the sun gear using equally spaced planets [31]. Vibration separation involves extracting a subset of a measured acceleration signal over a period of time when a given planet is aligned with a ring-mounted accelerometer. This extraction is then used to synthesize a new signal, termed the Vibration Separation Waveform (VSW), that represents a portion of the dynamics of the target gear. The underlying presumption is that the extracted signals are dominated by the simultaneous meshing of the aligned planet's teeth with the ring and sun gears and that averaging can be used to expose the sought-after dynamics. The tooth engagement schedules are used to match extracted data with each of the target gear's teeth.

Detecting damage on planet gears using vibration separation has been shown to be successful [29, 32]. However, detecting damage on the sun gear continues to be a challenge since its dynamics need to be assessed through the planet gears introducing a higher degree of complexity. Thus, there is a need to minimize planet dynamics while emphasizing sun dynamics.

Figure 1 gives a flowchart showing the three stages of the vibration separation processes used in this research. The Acquisition/Interpolation stage encompasses the simultaneous data collection of the vibration signal and the once-per-revolution tachometer signal of the target shaft. Using the tachometer signal, the data is partitioned into individual cycles. Each cycle is optionally filtered to remove known frequencies and interpolated to $\bar{N}$ points and then stored as a column in the Interpolation Matrix. This two-dimensional matrix has length, $\bar{N}$ and width, $N_{\text {extract }}$, which depends on the number of complete cycles in the measured data. Using a parameter called the hunting tooth ratio $\left(N_{T R}\right)$, to be explained later, columns are collected into HT Groups.

The next step is the Extraction stage. Each HT Group represents a full carrier cycle and within each cycle there is a point, $N_{i j}^{*}$, called the extraction index, which corresponds to when 
Acquisition \& Interpolation

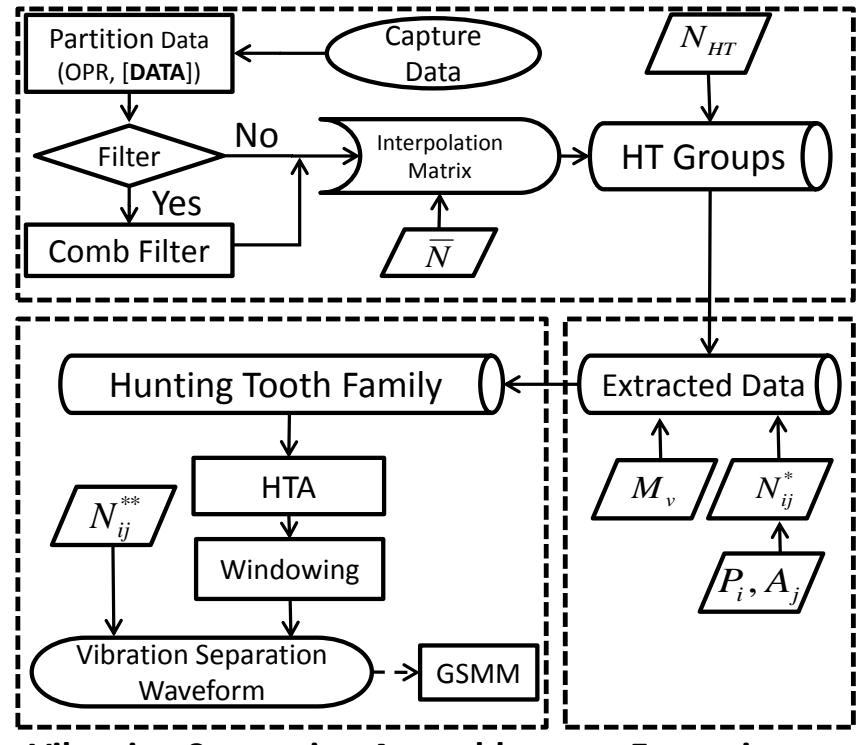

Vibration Separation Assembly

FIGURE 1. Vibration Separation Flow Chart

planet $P_{i}$ and accelerometer $A_{j}$ are aligned. Given the extraction index, $N_{i j}^{*}$, and a pre-determined number of tooth mesh periods, $M_{v}$, data is extracted and stored in a data structure called the Hunting Tooth Family (HT Family). The Hunting Tooth Family is a 5 dimensional data structure which contains a record for each accelerometer / planet combination with each record containing a 3D matrix. Each 3D matrix has rows, columns, and layers corresponding to rotation angle, carrier cycles, and HT Group respectively.

The next stage is Vibration Separation Assembly. In this stage, the waveforms within each HT Group are averaged producing a Hunting Tooth Average (HTA). The resulting waveforms are windowed and assigned a mapping index, $N_{i j}^{* *}$, that depends on the planet or sun tooth engaged at the time the data was extracted. The HTAs are then concatenated to form the Vibration Separation Waveform in an order determined by $N_{i j}^{* *}$. This approach applies to individual planets $(P G V S)$ as well as the sun gear $(S G V S)$. Since only one accelerometer and one planet is used, this technique is referred to as a single accelerometer / single planet $(S A S P)$. Another technique is GSMM. While not technically vibration separation, as will be shown later, it can still be developed within the same framework.

\section{Acquisition and Interpolation}

A schematic of a planetary gear indicating the locations of planets $\left(P_{i}\right)$ and accelerometers $\left(A_{j}\right)$ is given in Figure 2. The values differ for both research program and is given in Table 1.

Since the carrier rotates counter-clockwise, the planet num-

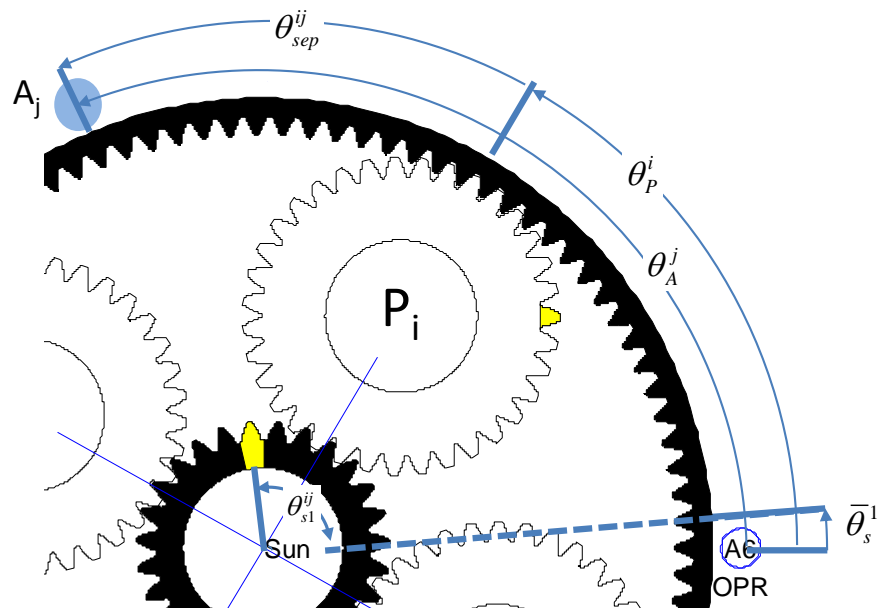

FIGURE 2. OH58C Planet/Accelerometer Layout

TABLE 1. Accelerometer and Initial Planet Orientation (deg.)

\begin{tabular}{lllllllll}
\hline & $A_{1}$ & $A_{2}$ & $A_{3}$ & $A_{4}$ & $A_{5}$ & $A_{6}$ & $A_{7}$ & $A_{8}$ \\
\hline UMD & 258 & 273 & 310 & 119 & 130 & $\mathrm{x}$ & $\mathrm{x}$ & $\mathrm{x}$ \\
\hline OSST & 112.7 & 87.23 & 61.81 & 36.36 & 10.91 & 0 & 294.55 & 247.23 \\
\hline \hline & $P_{1}$ & $P_{2}$ & $P_{3}$ & $P_{4}$ & (Pinion) & & \\
\hline UMD & 319.3 & 227.9 & 139.3 & 47.9 & 258 & & & \\
\hline OSST & 218.2 & 129.6 & 38.18 & 309.61 & 247 & & & \\
\hline
\end{tabular}

bering is clockwise, so that increasing planet number corresponds with the order in which they reach each accelerometer. The accelerometers are numbered counter-clockwise. The convention used is such that, when the planetary system is in its initial orientation at $\theta_{c}=0$, all planet teeth engaged with the ring gear are assigned $P_{i}^{1}$ and increase in the direction opposite to the gear's rotation. In addition, the sun gear's tooth closest to the origin is assigned $S T 1$ and has an initial angle given as $\overline{\theta_{s}^{1}}$.

During testing, data is collected and a tachometer provides the once-per-revolution pulse used to parse the signals into the individual carrier cycles. Since the first and last cycles are incomplete, they are rejected.

Interpolation converts the points from the temporal domain to the angular domain and is used to to ensure that all cycles have measurement points at the same angular position. The number of interpolation points, $\bar{N}$, is chosen to satisfy 3 requirements:

1.) $\bar{N}$ is close to the average number of points representing one carrier cycle. $\left(\bar{N}_{\text {avg }}\right)$.

2.) $\bar{N}$ can be divided into $N_{r}$ equal sections. (It is convenient to work in units associated with a single tooth mesh period (TMPs) consisting of $N_{T P}$ points.)

3.) $\bar{N}$ is odd. This simplifies the signal reconstruction. 
The smallest integer value, $N_{T P}$, is found by solving Eq. 1 iteratively.

$$
N_{T P}=\min _{N_{T P}}\left(\left|N_{r}\left(N_{T P}-1\right)+1-\bar{N}_{a v g}\right|\right)
$$

Once $N_{T P}$ is solved, $\bar{N}$ is determined from Eq. 2

$$
\bar{N}=N_{r}\left(N_{T P}-1\right)+1
$$

The Vibration Separation Waveform consists of either $N_{p}$ $T M P s$ of an individual planet gear or $N_{s} T M P s$ of the sun gear. The number of points used to construct their corresponding Vibration Separation Waveform, $\ell_{v}$, is given by:

$$
\ell_{v}=N_{p, s}\left(N_{T P}-1\right)+1
$$

where $N_{p, s}$ is either $N_{p}$ or $N_{s}$.

\section{Extraction}

The data extracted from each carrier cycle depends on three parameters: planet gear angle $\theta_{P}^{i}$, accelerometer angle, $\theta_{A}^{j}$, and the number of data points to extract, $\ell_{E}$. Since the first carrier cycle is rejected, the analyzed portion of the data starts at $\theta_{c}=$ $2 \pi$.

The spacing between planets on the $\mathrm{OH}-58 \mathrm{C}$ is not equal. In particular, the planets have an ' $\mathrm{X}$ ' orientation, a given planet is closer to one of its immediate neighbors. The planet separation angle must be an integer multiple of the least mesh angle given by $\lambda=\frac{2 \pi}{N_{s}+N_{r}}$. For the OH-58C, $\lambda=\frac{\pi}{63}$ and the ring gear pitch is $P_{b}=\frac{2 \pi}{99}$. For 4 planets, the spacing is either $31 \lambda$ or $32 \lambda$, not an integer multiple of $P_{b}$, therefore the planets are not in phase [33].

Unequal spacing results in data extractions dependent on which planet reaches the target accelerometer first. If $P_{1}$ or $P_{3}$ is first, the angular sequence is $\left[0 \frac{32 \pi}{63} \pi \frac{95 \pi}{63}\right]$, otherwise, the sequence is $\left[0 \frac{31 \pi}{63} \pi \frac{94 \pi}{63}\right]$. Finally, the condition to test if the planets are sequential is $\sum_{i=1}^{4} N_{r} \Psi_{i}=m \pi$, where $\Psi_{i}$ is the angle for each planet starting with $\Psi_{1}=0$ and $m$ is an integer. Using $\Psi=\left[0 \frac{32 \pi}{63} \pi \frac{95 \pi}{63}\right]$ as an example results in $(2+64 / 63) \pi \neq m \pi$ and reveals that the $\mathrm{OH}-58 \mathrm{C}$ is also non-sequential. This means that each planet is operating at a different point along the pressure line; thus, the sudden changes in stiffness occur at different times [34].

For a given initial planet angle, $\theta_{P}^{i}$, and accelerometer angle, $\theta_{A}^{j}$, the initial carrier angle separating the two is given by:

$$
\theta_{\text {sep }}^{i j}=\bmod 1\left(\theta_{A}^{j}-\theta_{P}^{i}, 2 \pi\right)
$$

where $\bmod 1(x, y)=\bmod (x-1, y)+1$ is the one-based modulo function. This is done to ensure that the counter clockwise angle is used for $\theta_{s e p}^{i j}$, which corresponds to the rotating direction of the carrier. Since the carrier cycle is interpolated to $\bar{N}$ equally spaced points, each spacing represents $\Delta \theta_{c}=\frac{2 \pi}{\bar{N}-1}$. Therefore the closest index point corresponding to when $P_{i}$ is aligned with $A_{j}$ is given by:

$$
N_{i j}^{*}=\left\lfloor\frac{\theta_{\text {sep }}^{i j}}{\Delta \theta_{c}}\right\rceil
$$

Table 2 gives $\theta_{\text {sep }}^{i j}$ for the two configurations. With four planets

TABLE 2. Separation Schedule $\left(\theta_{\text {sep }}^{i j}\right)$ (degrees)

\begin{tabular}{lllllllll}
\hline UMD & $A_{1}$ & $A_{2}$ & $A_{3}$ & $A_{4}$ & $A_{5}$ & & & \\
\hline$P_{1}$ & $\mathbf{2 9 8 . 7}$ & 313.7 & 350.7 & 159.7 & 170.7 & & & \\
$P_{2}$ & $\mathbf{3 0 . 1}$ & 45.2 & 82.2 & 251.2 & 262.2 & & & \\
$P_{3}$ & $\mathbf{1 1 8 . 7}$ & 133.7 & 170.7 & 339.7 & 350.7 & & & \\
$P_{4}$ & $\mathbf{2 1 0 . 1}$ & 225.2 & 262.2 & 71.2 & 82.2 & & & \\
\hline OSST & $A_{1}$ & $A_{2}$ & $A_{3}$ & $A_{4}$ & $A_{5}$ & $A_{6}$ & $A_{7}$ & $A_{8}$ \\
\hline$P_{1}$ & 254.5 & 229 & 203.6 & 178.2 & 152.7 & 141.8 & 76.4 & 29 \\
$P_{2}$ & 343.1 & 317.6 & 292.2 & 266.7 & 241.3 & 230.4 & 164.9 & 117.6 \\
$P_{3}$ & 74.5 & 49 & 23.6 & 358.2 & 332.7 & 321.8 & 256.4 & 209 \\
$P_{4}$ & 163.1 & 137.6 & 112.2 & 86.7 & 61.3 & 50.4 & 344.9 & 297.6 \\
\hline
\end{tabular}

and 5 (UMD) or 8 (OSST) accelerometers, there are 20 or 32 extraction locations within each carrier cycle, respectively.

Letting $M_{v}$ be an odd integer representing the number of $T M P s$ of data desired, the corresponding number of points extracted is given by:

$$
\ell_{E}=M_{v}\left(N_{T P}-1\right)+1
$$

The constraint that $M_{v}$ is an odd integer ensures the waveform associated with $A_{i} / P_{j}$ has a distinct midpoint.

As gears mesh, the hunting tooth ratio $\left(N_{H T}\right)$ governs the rate at which a pair of gear teeth meet. It is given by the smaller of the least common, non-unity, factor between the number of teeth on a pair of gears, or the smallest number of teeth. As an example, for the systems under study, the hunting tooth ratio between the number of planet teeth, $N_{p}=35$, and ring gear teeth, $N_{r}=99$, is $N_{H T}=N_{p}=35$. This means that for a given ring gear tooth, it takes 35 carrier cycles before a given planet tooth meets that ring tooth again. Even though the sun does not mesh directly with the ring gear, their hunting tooth ratio of three still plays an important role. For every three carrier cycles, a given 
sun gear tooth will align with a given ring gear tooth. This has consequences on how the extractions are handled and allows time synchronous averaging to be performed, but on cycles spaced at the hunting tooth ratio.

Within the first consecutive $N_{p}$ cycles, every planet tooth aligns with a given accelerometer once. The collection of these cycles constitute a set. Likewise, each additional set of $N_{H T}$ cycles creates another set. There are $N_{\text {set }}=\left\lfloor\frac{N_{\text {extract }}}{N_{H T}}\right\rfloor$ sets available where $N_{\text {extract }}$ is the number of carrier cycles contained in one data set. Similar cycles of different sets can be combined into HT Groups. This is akin to grouping cycles spaced at the hunting tooth ratio and takes the following sequence form:

$$
\text { HT Group } x:\left\{x, x+N_{H T}, x+2 N_{H T} \ldots, x+\left(N_{\text {set }}-N_{H T}\right)\right\}
$$

for $x \in\left\{1, \ldots, N_{H T}\right\}$.

The extraction process is illustrated in Figure 3. There are three HT Groups shown: 1st, 2nd, and last. Each group consists of multiple waveforms according to Equation 7. The four boxes in each HT Group represent the extraction regions centered on $N_{i j}^{*}$ and only needs to be determined once for each $P_{i} / A_{j}$ pair. The width of the box represents the length of data extracted, $\ell_{E}$.

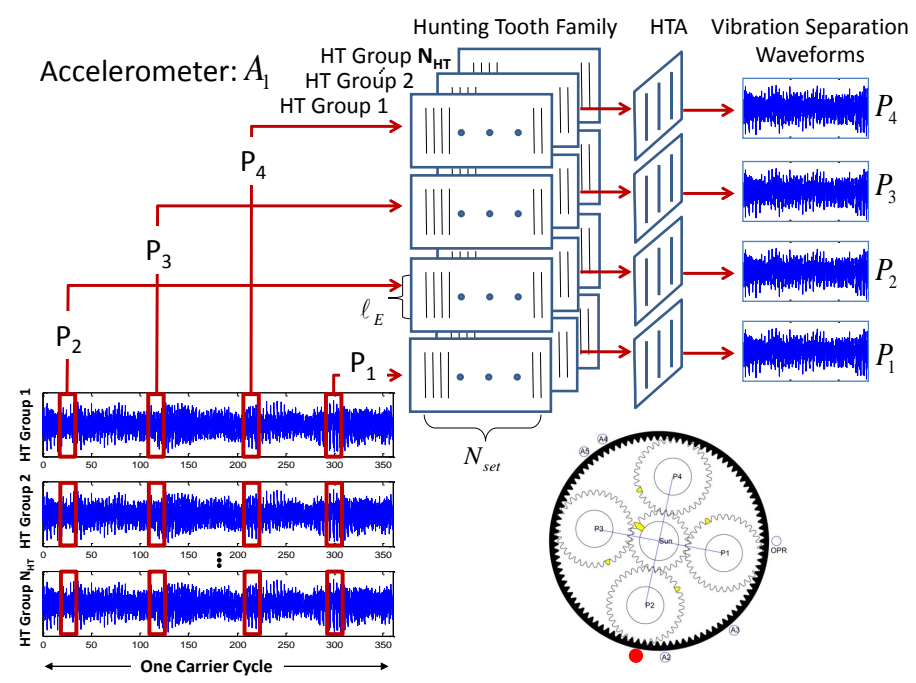

FIGURE 3. Vibration Separation Extraction Example. $A_{1}$

For this case (in bold, Table 2), planet $P_{2}$ is the first planet to reach $A_{1}$. For HT Group 1 , this collection of $N_{\text {set }}$ waveforms are assigned to $P_{2}$ and make up a portion of the first layer in the Hunting Tooth Family. The next planet to arrive at $A_{1}$ is $P_{3}$. These extracted waveforms are assigned to $P_{3}$ and also assigned to the first layer. This continues for $P_{4}$ and $P_{1}$ completing the first Hunting Tooth Family layers associated with HT Group 1. The same is done for HT Group 2, where the assignments make up a second layer. The total number of layers in the Hunting Tooth Family is $N_{H T}$. Each HT Group layer is assigned to a tooth $\left(M_{v}=1\right)$ or span of teeth $(M v>1)$.

\section{Vibration Separation Assembly}

The waveforms in each layer of the Hunting Tooth Family are averaged together producing a time synchronously averaged signal called the Hunting Tooth Average (HTA). These final HTAs are then used to synthesize the Vibration Separation Waveform by placing each HTA at a position determined by the mapping index, $N_{i j}^{* *}$, which is governed by the planet's tooth mesh schedule. If $M_{v}>1$, overlapping of data will occur. Windowing is used to merge overlapping data to reduce distortion at the edges [29,32].

\section{SGVS - GENERAL}

Sun Gear Vibration Separation is challenging because isolating dynamics associated with a given sun gear tooth through a simultaneously meshing planet gear involves multiple stages of dynamics. Figure 4 shows the 27 tooth sun gear with teeth numbered clockwise. Each plot contains markings at distinct angles used as symbolic representations of the region of the sun gear that is considered aligned as a planet passes $A_{1}$. The simultaneously aligned planets are denoted by the three Planet IDs that accompany the markings and the sequence extends radially inward in the planet passing order. In addition this shows that for the $\mathrm{OH}-58 \mathrm{C}$, if the hunting tooth ratio between the sun gear and the ring gear is not equal to $N_{s}$, only a fraction of the teeth are aligned with a given accelerometer when data is extracted. For PGVS, since $N_{H T}=N_{p}$, extractions are possible when each planet tooth is in the same physical orientation. This allows for each individual tooth mesh waveform to be directly compared producing highly correlated waveforms. However, for the current $S G V S$ scenario, $N_{H T}=3$. Therefore only 3 teeth can be directly compared if 'seen' through a single planet. For example, this corresponds to regions between ST5/ST6, ST23/ST24, and ST14/15 in Figure 4 and using $P_{1}$. Thus, a direct tooth-to-tooth comparison for all teeth is not possible.

The markings in Figure 4 correspond to $M_{v}=1$. To address this downfall, data is extracted representing a larger number of TMPs $(M v>1)$. Since $N_{H T}=3$, a span of $M_{v}=\frac{N_{s}}{N_{H T}}=9$ teeth is chosen. Each time a planet passes an accelerometer, the extracted data corresponds to four TMPs prior to and 4 TMPs after planet/accelerometer alignment. For this case, there is no overlapping therefore a uniform window will suffice. 


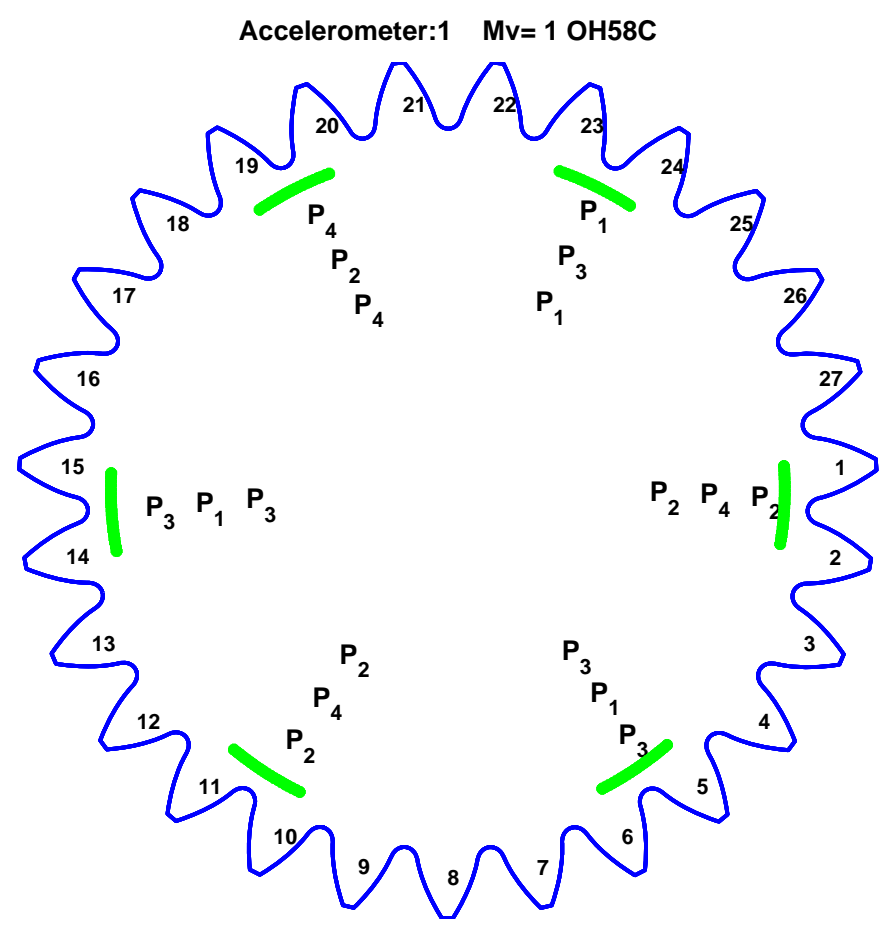

FIGURE 4. Sun Gear's Aligned Region $M_{v}=1$ and $A_{1}$

\section{Sun Tooth Mesh Schedule}

In order to match extracted dynamics with the correct sun tooth region, planetary indexing is used to create the sun tooth meshing schedule. As the carrier rotates $m$ revolutions, the location of $S T 1$ is tracked. In order for $P_{i}$ to align with $A_{j}$ during cycle $m$, the carrier must rotate $\theta_{\text {sep }}^{i j}+m(2 \pi)$ radians. The sun gear rotates in the same direction at an angle given by:

$$
\theta_{s}=\left(1+\frac{N_{r}}{N_{s}}\right)\left(\theta_{s e p}^{i j}+2 \pi m\right)
$$

using the identity: $\theta_{s}=\left(1+\frac{N_{r}}{N_{s}}\right) \theta_{c}$.

The local angle of ST1 is given by:

$$
\theta_{s 1}^{i j}=\bmod 1\left\{\left(1+\frac{N_{p}}{N_{r}}\right)\left(\theta_{\text {sep }}^{i j}+2 \pi m+\overline{\theta_{s}^{1}}\right), 2 \pi\right\}
$$

Using the clockwise angle to $A_{j}$, corresponding to increasing Sun Tooth ID number, the Sun Tooth ID number is given by

$$
S T n=\left\lfloor\frac{\left.\bmod 1\left(\theta_{s 1}^{i j}-\theta_{A}^{j}\right), 2 \pi\right)}{\Delta \theta_{s}}\right\rceil+1 .
$$

Table 3 shows the tooth meshing schedule in term of the approximate tooth ID using $M_{v}=9$ for the UMD configuration. Since the sun tooth pattern repeats after 3 cycles, the Table 3 represents a complete set. Sun Tooth ID values are used instead of angular values to make the tables more intuitive. However, during implementation, the actual angles are used. Three HT Groups are used to create the sun gear's Vibration Separation Waveform. There are 15 sub blocks representing different HT Group / Accelerometer combinations. The highlighted columns within each sub-block represent the assigned Tooth ID. Each row of each sub block contains $M_{v}=9$ Tooth ID numbers per planet pass.

Figure 5 is a graphical representation of the first sub-block of Table 3 for which the gray sun tooth represents ST1 and the black tooth is the one indicated in the table.
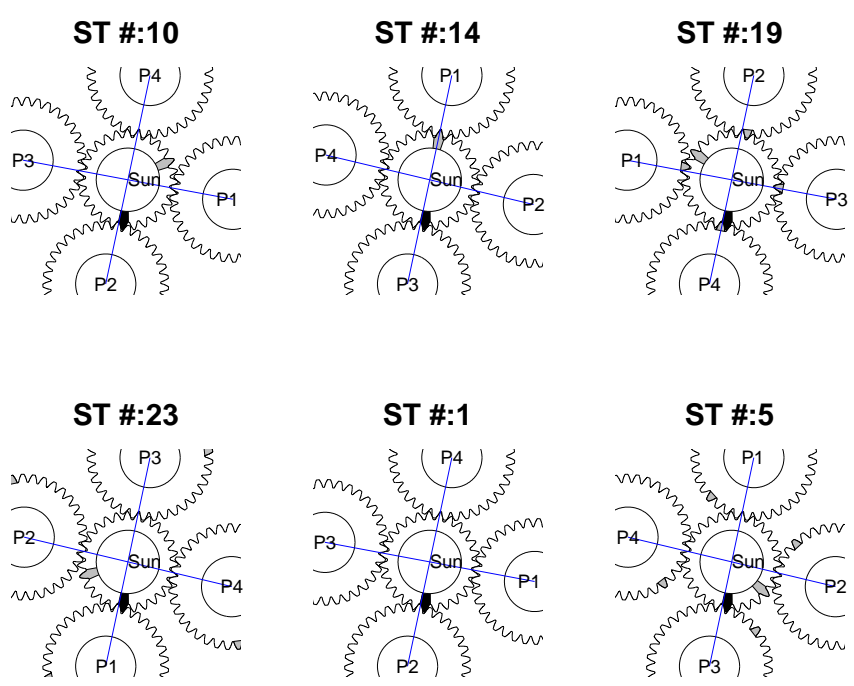

FIGURE 5. Sun Meshing Positions (HT Group 1, $A_{1}$ )

When $P_{2}$ aligns with $A_{1}$, the sun gear is in mesh between ST10 and ST11. The chart's value of ST10 is chosen for clarity. Data is extracted that corresponds to when ST6 through ST14 are in mesh with $P_{2}$ as $P_{2}$ passes $A_{1}$. This is then repeated when the next planet, $P_{3}$, arrives at $A_{1}$ and data associated with teeth ST10 to ST18 are extracted. As shown in Table 3, after 6 planet passes ( 1.5 cycles), the sequence repeats.

\section{SUN GEAR VIBRATION SEPARATION - SASP METHOD}

The aforementioned discussion provides the necessary background to the development of the $S A S P$ method for vibration separation. Since $N_{H T}=3$, there are 3 HT groups, each associated with a range of sun teeth. For example, extractions associated with $A_{2} / P_{3}$ are highlighted in Table 3 . HT Group 1 is associated 
TABLE 3. Sun Tooth Meshing Schedule OH-58C $M_{v}=9$ (UMD)
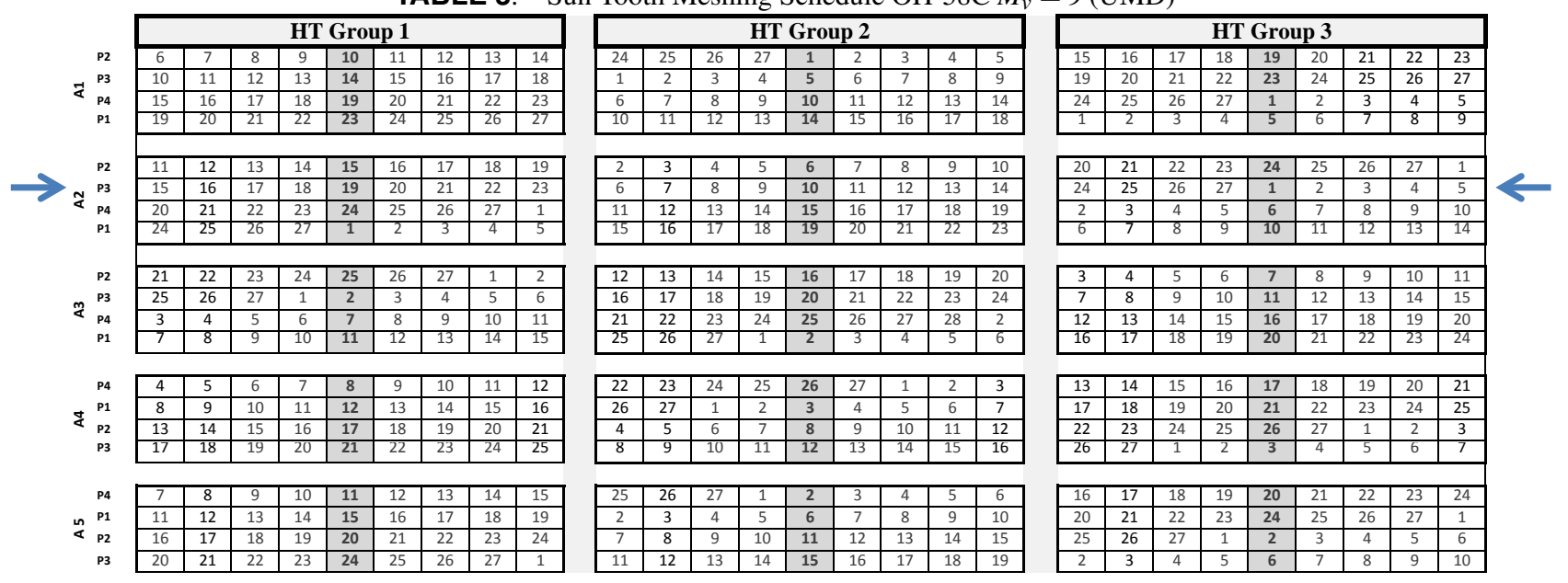

with ST19, HT Group 2 is associated with ST10, and HT Group 3 is associated with ST1. The Vibration Separation Waveform is synthesized by computing the HTA of each HT Group and combining the resulting waveforms in order of monotonically increasing Tooth ID which reflect the following order: $\{\overline{H T 3} \overline{H T 2}$ $\overline{H T 1}\}$ One Vibration Separation Waveform is created for each accelerometer/planet combination.

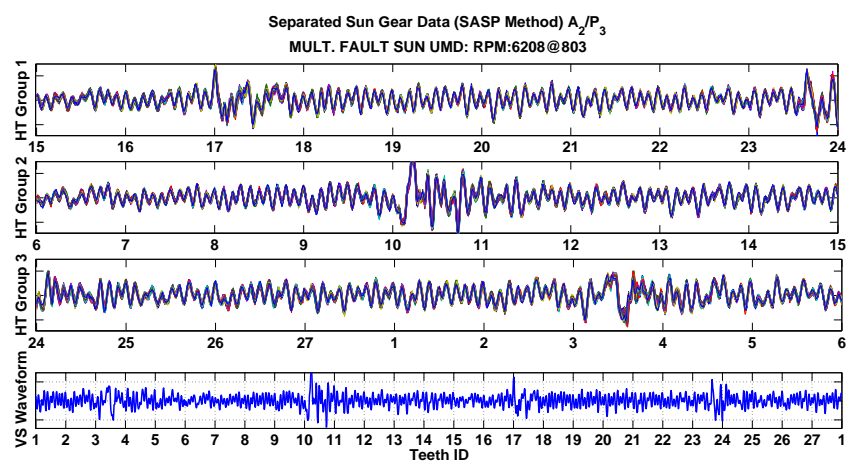

FIGURE 6. SGVS Example Output $A_{2} / P_{3}$

The plot in Figure 6 shows an example Hunting Tooth Family. The cycles associated with HT Group 1 are plotted together on the first row. The same is true for HT Group 2 and HT Group 3 . The $x$-axis represents the sun tooth angle, but has been replaced by Sun Tooth IDs to make the plot more intuitive. A high degree of correlation is observed within each HT Group giving confidence that the finer waveform details are being enhanced. The fourth row is the Vibration Separation Waveform.

\section{GEOMETRICALLY SYNCHRONIZED MEASUREMENT METHOD}

Unlike vibration separation, which uses the premise that the engaged teeth closest to the accelerometer dominates the signal, Geometrically Synchronized Measurement Method (GSMM) attempts to characterize all engaged teeth as a whole. The method described in this section presumes that if a gear tooth is damaged, when engaged, it produces a distinctive vibration signature at all accelerometers. As discussed, the transmission's vibrations spectrum is complicated by the simultaneously meshing gears, and often the details associated with a given component are obscured, even after time-synchronous averaging. Because of the extra experimental effort to implement vibration separation such as planetary indexing, and component locking during overhauls, an alternative method to transmission diagnostics was made possible [29]. This method exploits the geometric synchronization between the sensors and planetary geometry.

The technique relates subsets of a representative vibration signal to the group of sun teeth in mesh, called mesh groups. Figure 7 shows an enlarged view of the sun gear region with the current mesh group (ST4,ST24,ST18,ST11) highlighted.

At any given time, the sun gear is in mesh with all planets. If the planets were equally spaced, then the same set of four teeth would always be in mesh together, allowing the creation of constant mesh groups. However, due to the non-sequential nature of the $\mathrm{OH}-58 \mathrm{C}$ as discussed earlier, the spacing between consecutive meshed regions is not constant, but instead exhibits a 6/7/7/7 pattern. Therefore, a more refined approach is used. The meshed regions are defined using the lines of centers between the sun and planets as references. The centers of each planet are tracked during meshing and then mapped to regions on the sun tooth. This collection of sun tooth angles at any given time make up a mesh group instead of the discrete Tooth IDs.

The GSMM works by stepping through the representative 


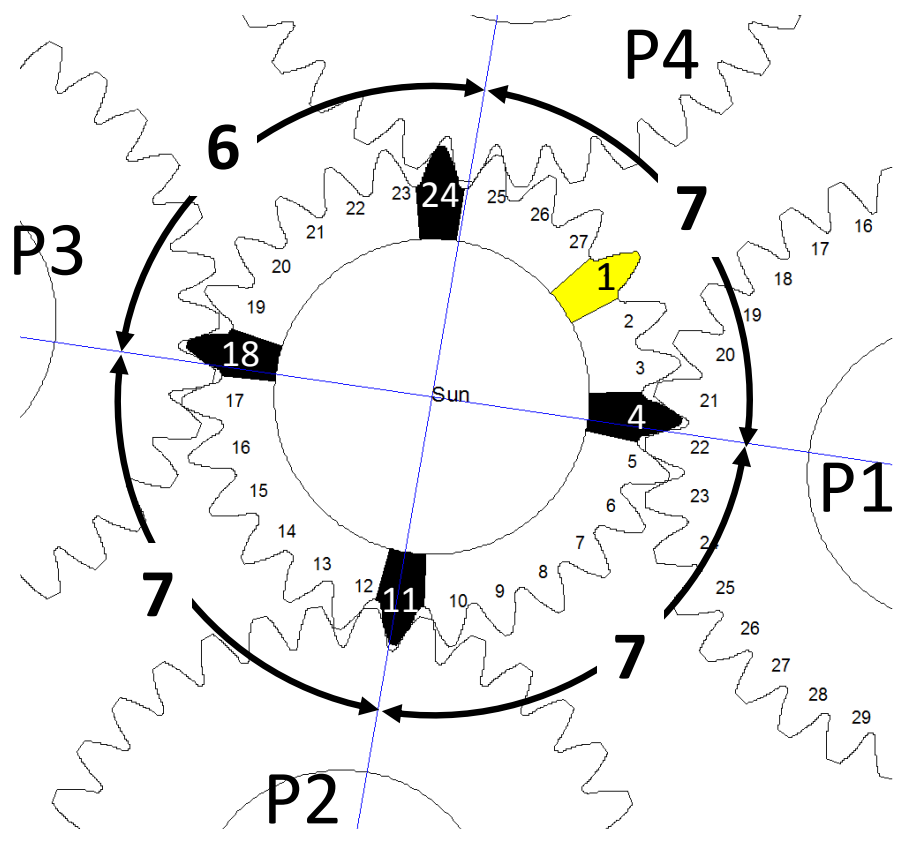

FIGURE 7. Mesh Groups (OH-58C)

signal in increments corresponding to a single planet TMP. This signal is synthesized from averages based on the first $N_{H T}$ cycles and concatenated in order. In this manner, this technique can be envisioned within the framework of Vibration Separation for the special case of $M_{v}=N_{r}$. For the sun gear with $N_{H T}=3$, this results in a 3 cycle signal with the form: $\{\overline{H T 1} \overline{H T 2} \overline{H T 3}\}$ where the bar represents the HTA. At each step, a condition indicator (CI ) is calculated on the subset of data and the value is assigned to the sun teeth in mesh. A circular histogram displays the tallied contributions of each tooth. The expected result is a uniform bar radius for the non-damaged case and a mesh group pattern consisting of 4 bins, separated in the $6 / 7 / 7 / 7$ pattern for the damage cases. The Tooth ID numbering is clockwise with ST1 positioned at zero degrees. A typical display is given in Figure 8.

Since spalls are known to produce pulses in the measured signal, a CI that emphasizes the outliers is sought. The following CI is used: $C I=\frac{1}{N_{T P}} \sum_{i} x_{i}^{6}$. The plots are presented normalized to unity since it is only the relative values that are important.

\section{EXPERIMENTAL SETUP}

The tests were performed in the NASA Glenn 500-hp helicopter transmission test facility which has been used by many researchers to advance the field of helicopter HUMS. The OH$58 \mathrm{C}$ transmission is rated at maximum continuous power of 335 $\mathrm{HP}$ at $6180 \mathrm{RPM}$ input speed. The main-rotor transmission is a two stage reduction gearbox with an overall reduction ratio of 17.44:1. The first stage is a spiral bevel gear set with a 19-tooth pinion that meshes with a 71 tooth gear. The seconds is a plane-
Baseline UMD: 6060RPM@3099

Accelerometer: 4

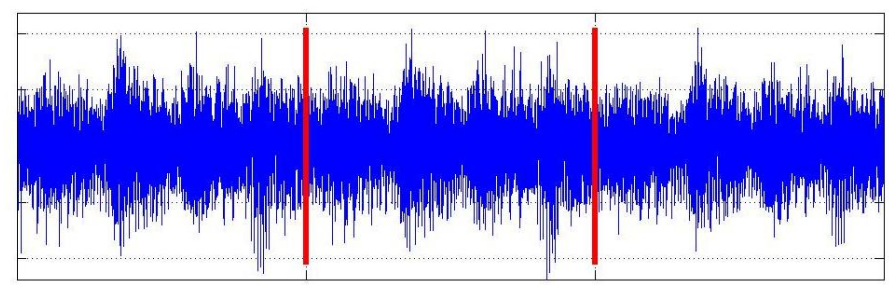

HT1--HT2--HT3

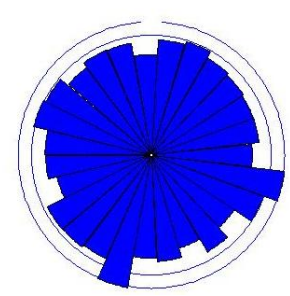

FIGURE 8. Sample Output - GSMM

tary set with a 27-tooth sun, 35-tooth planet, and a fixed 99-tooth ring gear. The bevel-gear shaft is connected through a spline to the sun gear shaft. The rotor mast is splined to the carrier.

Three seeded fault cases were tested at input speeds around 6000 RPM with torques ranging from $25 \%$ to $100 \%$. All measurement discussed were conducted at full mast loading. Tachometers were used on both the input and output shafts. The output tachometer signal is used to partition the measured data into individual carrier cycles as well as count them to produce a reset pulse indicating when the planetary geometry has returned to its initial orientation. The planetary system has a repeat cycle of 105 carrier cycles.

\section{Accelerometer Placement}

The initial planetary orientation, number of accelerometers, and the accelerometer locations differed between the two test programs as shown in Figure 9.

UMD: Accelerometer $A_{1}$ was mounted to the input end of the transmission just above the pinion. The input spiral bevel pinion has a thrust load against its tri-plex ball bearing and single roller bearing. This is the direction of $A_{1}$ 's sensitivity axis and is aligned to the rotational axis of the input shaft [35]. Accelerometer $A_{2}$ is positioned 15 degrees counter-clockwise from $A_{1}$ and mounted on the side of the transmission housing. Accelerometers $A_{3}$ and $A_{5}$ are mounted to a special bracket and are located 180 degrees apart. This is the same bracket and location used in flight tests [36,37]. Accelerometer $A_{4}$, like $A_{2}$, is mounted to the side of the transmission housing. All accelerometers have their sensitivity axis in the transmission's radial direction as this direction provides the best results [38]. 


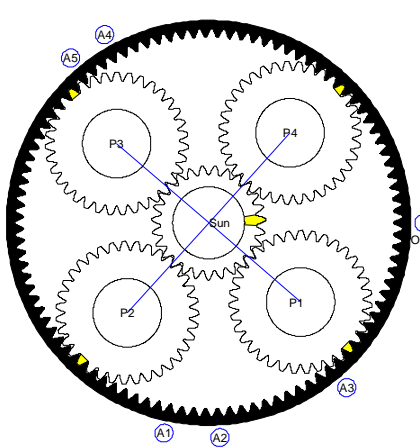

(a)

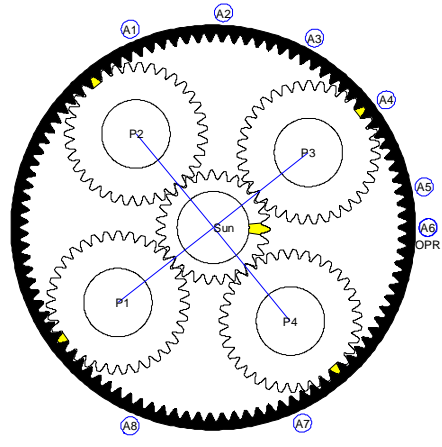

(b)
FIGURE 9. Initial Orientations $\left(\theta_{c}=0\right)$ a) UMD b) OSST

OSST: Eight piezoelectric accelerometers were installed. Six accelerometers $(1,2,3,4,5$, and 7) were directly mounted to the transmission housing adjacent to the ring gear through threaded holes. The holes were tapped in the housing at various positions along the circumference of the ring gear to measure vibration in the radial direction relative to the planetary. Two accelerometers (6 and 8) were mounted on brackets installed on the transmission top cover housing to measure vibration in the tangential direction relative to the planetary. For accelerometer 8 , the bracket was mounted directly to the transmission housing. For accelerometer 6 , the bracket was mounted on a top cover mounting stud. Accelerometers 1 through 5 were spaced equally at 7 ring gear teeth and number in the counter-clockwise direction.

The accelerometers had a bandwidth of 50kHz. The outputs of the accelerometers and tachometer pulses were routed to antialiasing filters and a PC-based data acquisition system. For all test cases and test conditions, data were acquired at $50 \mathrm{kHz}$ sampling rate with a $25 \mathrm{kHz}$ aliasing filter cut-off frequency. The data were acquired for $40 \mathrm{sec}$. per set.

\section{Test Components}

In total, there are two sets of baseline cases, two sets of single spall cases, one test for the multiple spall, and one test for the cracked sun gear. The single spall case is shown in Figure 10. This spall covers around $75 \%$ of the toothface. No other appreciable damage was noticed via visual inspection on any of the other teeth.

Another component tested was the sun gear shown in Figure 11. This gear had four teeth with severe damage. On ST10, a spall about one-third the facewidth exists. On tooth STID12, there is a chip at the tip which extends about $20 \%$ of the facewidth. ST14 has spall covering about $80 \%$ of the facewidth and, on ST15, almost the full facewidth is spalled.

A simulated crack was machined using Electrical Discharge

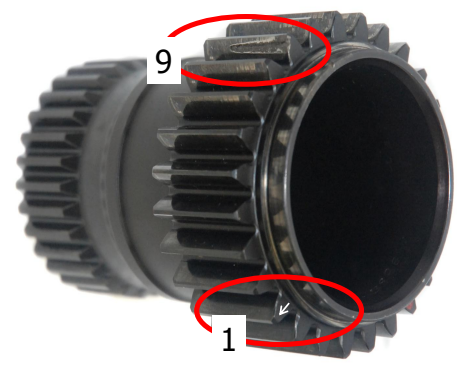

FIGURE 10. Sun Gear: Spalled Sun Gear Case

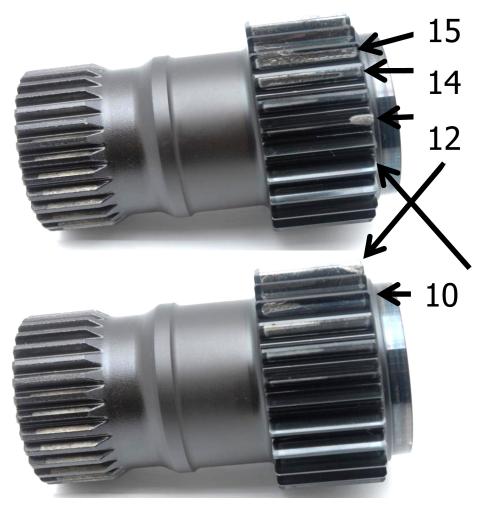

FIGURE 11. Sun Gear: Multiple Tooth Spall Case

Machining (EDM) and is shown in Figure 12. The notch was placed in the tooth fillet region along the complete width of the tooth. It had a crack depth of about 25 percent of the total tooth cross section length and a circular path similar to that which would naturally occur [39].

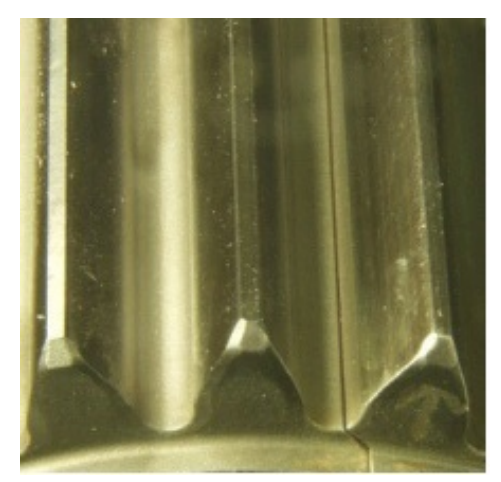

FIGURE 12. Sun Gear: 25 percent EDM root crack

During testing, the transmission ran continuously. Ten sets of data were collected with durations of either 20 or 40 seconds. Each acquisition was activated by a reset trigger indicating that 
the planetary orientation was in its pre-determined position.

\section{RESULTS}

\section{$S G V S$-SASP Method}

For the following plots, signals from $A_{4}$ (UMD) and $A_{1}$ (OSST) were chosen because they were farthest from the input pinion (Table 1). In addition, the reduced torque cases were chosen because they tended to provide better results.

Figures 13 and 14 show baseline results for tests conducted at 6294 RPM @ 1717 in-lbs. Figure 13 shows the individual HT Groups using accelerometer $A_{4}$ as 'seen' through planet $P_{1}$. Each HT Group represents a collection of 34 waveforms illus-

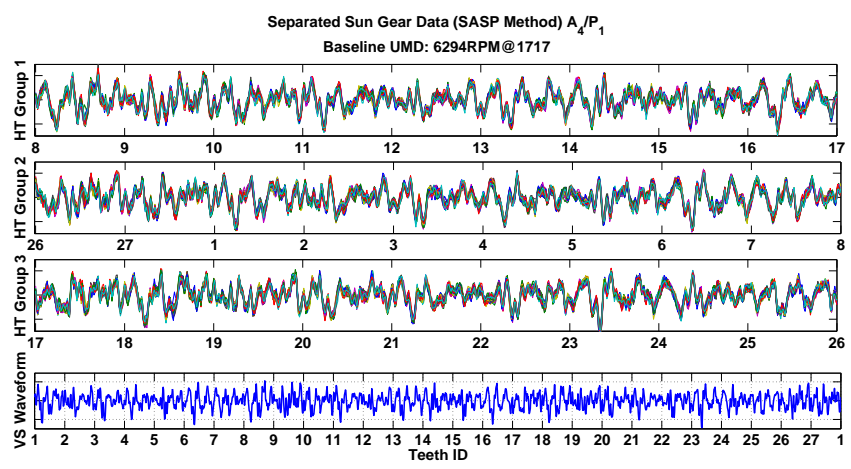

FIGURE 13. HT Groups: Baseline (UMD) - $A_{4} / P_{1}$

trating the high correlation between waveforms in the same HT Groups but not necessarily between different HT Groups. One of the key benefits of this technique is that averaging in HT Groups preserves the fine individual tooth mesh waveform details.

The Vibration Separation Waveform, as 'seen' through each planet using $A_{4}$, is shown in Figure 14. The HT Groups from Figure 13 were used to create the assembly of the first row.

Figures 15 and 16 show results from the single tooth spall case using the OSST results for the 6180 RPM @ 1708 in-lbs for $A_{1} / P_{1}$. Both figures reveal a distinct waveform associated with ST20 indicating that a fault is present.

Figures 17 and 18 shows the result for the 25 percent crack case for $6180 \mathrm{RPM} @ 1708$ in-lbs for $A_{1} / P_{1}$. It is difficult to visually detect the presence of the crack from inspections as no individual tooth mesh waveforms, or group of waveforms are distinct.

Figures 19 and 20 show the result for the multiple fault case for 6294 RPM @ 1717 in-lbs. In Figure 19, ST3, ST9, ST 16, and ST 23 have distinct individual tooth mesh waveforms. This set of teeth also form a mesh group revealing that waveform devi-

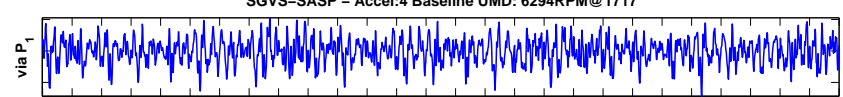

sity

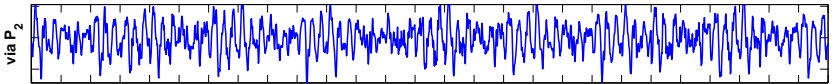

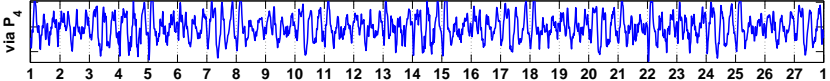

FIGURE 14. VSW via each $P_{i}$ : Baseline (UMD) - $A_{4}$

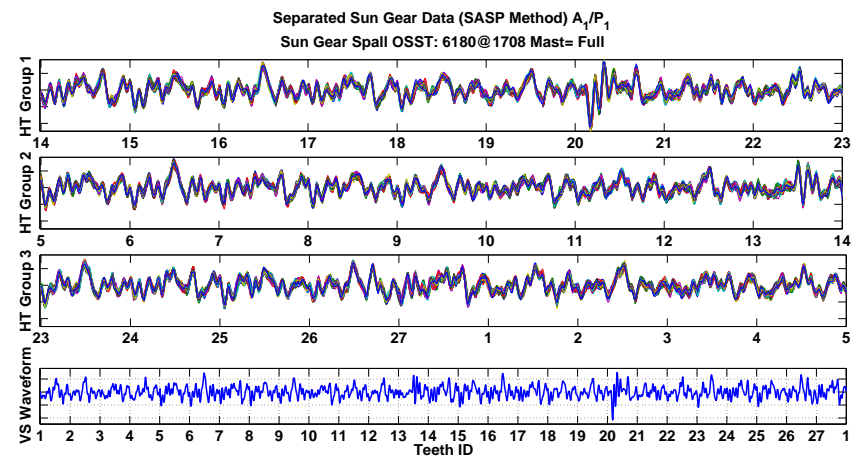

FIGURE 15. HT Groups: Single Spall (OSST) $-A_{1} / P_{1}$

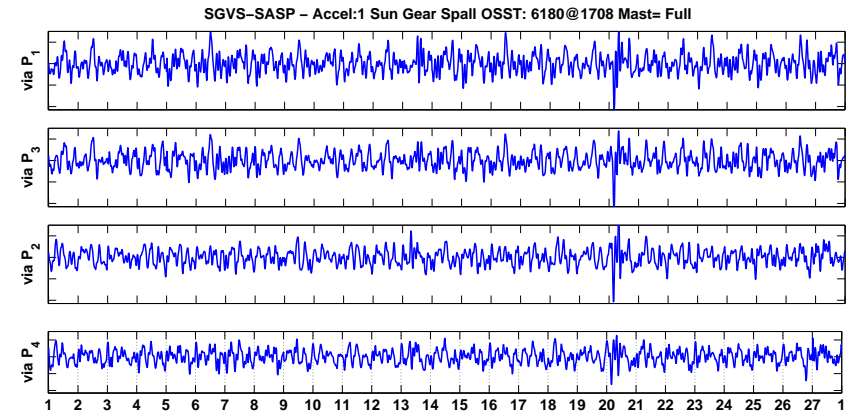

FIGURE 16. VSW via each $P_{i}$ : Single Spall (OSST) - $A_{1}$

ations in sun gear mesh groups are strong indicators of sun tooth damage.

\section{GSMM}

Figure 21 to Figure 24 show a schematic of the transmission on the left, the HTA signal along the top, and the circular histogram on the right. The signals used are $A_{4}$ for UMD and $A_{1}$ for 


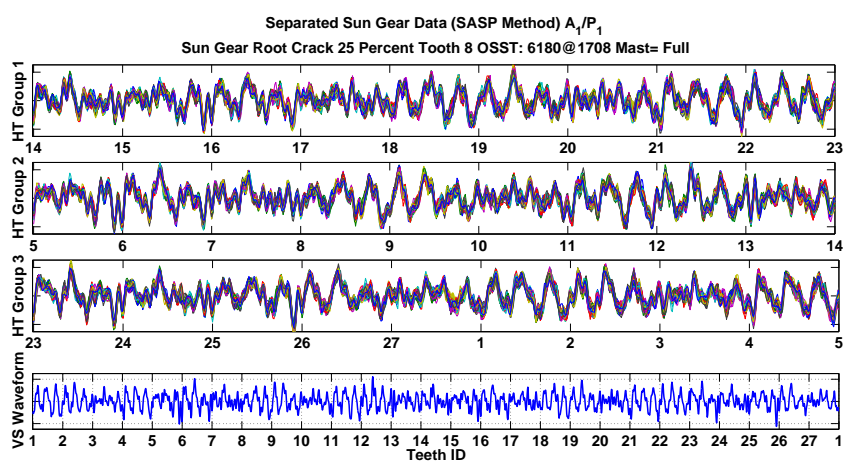

FIGURE 17. HT Groups: Crack - $A_{1} / P_{1}$

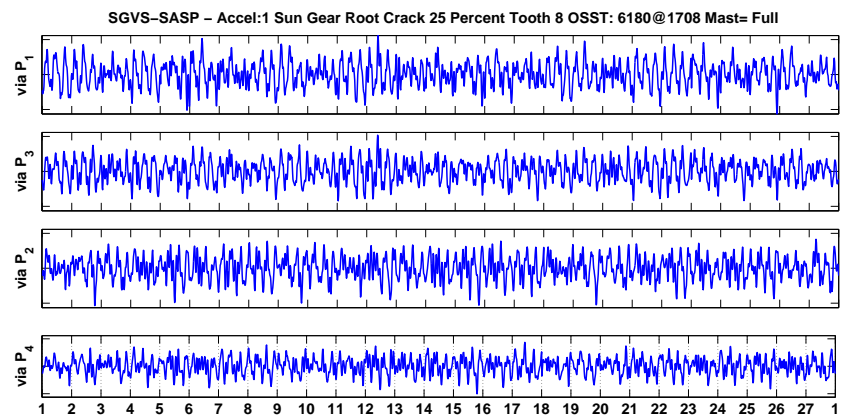

FIGURE 18. VSW via each $P_{i}$ : Crack - $A_{1}$

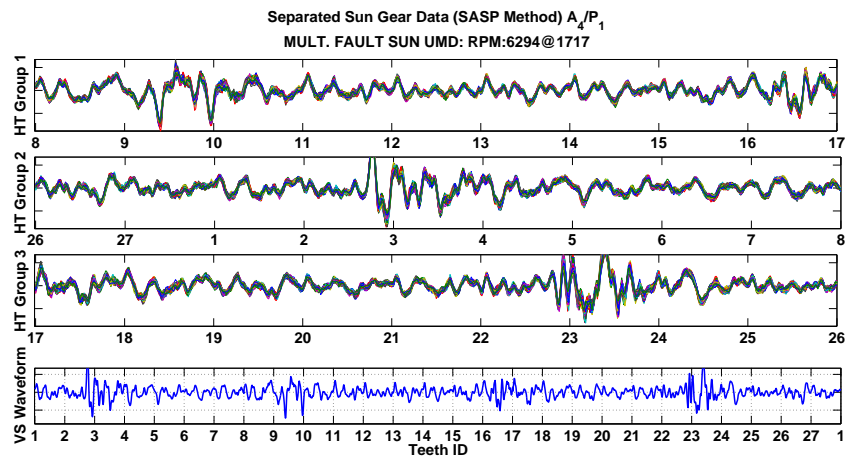

FIGURE 19. HT Groups: Multiple Spall (UMD) - $A_{4} / P_{1}$

\section{OSST.}

Figure 21 gives the results for the baseline case. The radius is normalized to unity and most of the bars uniform in radius suggesting that little bias exists due to damage. Figure 22 shows the case for a single tooth spall. A 4 bin, 6/7/7/7 pattern is evident and correctly indicates sun tooth damage. Figure 23 is the case

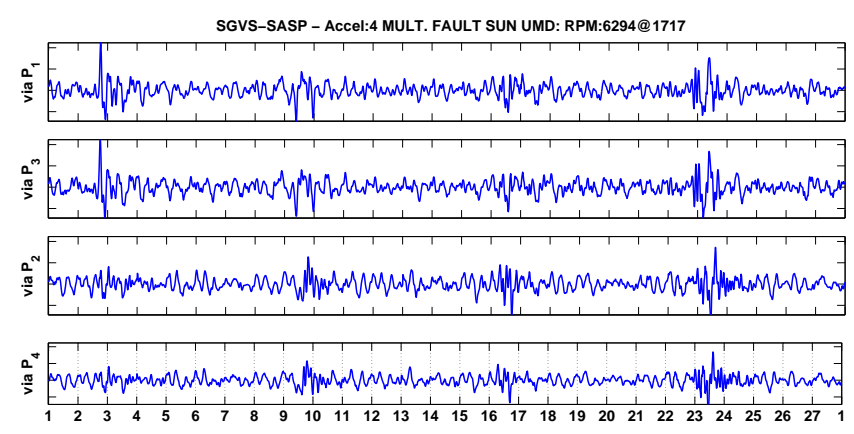

FIGURE 20. VSW via each $P_{i}$ : Multiple Spall (UMD) - $A_{1}$

Baseline UMD: 6294RPM@1717 Accelerometer: 4

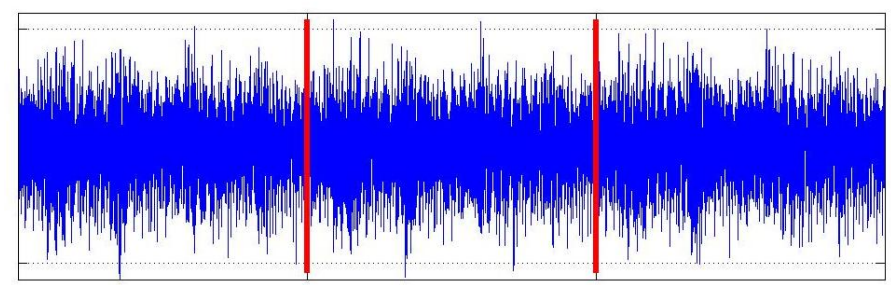

HT1--HT2--HT3

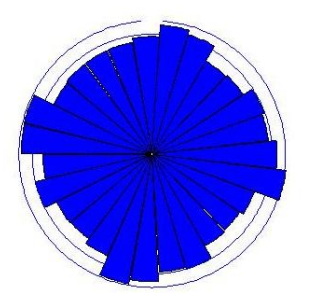

FIGURE 21. GSMM Baseline (UMD) $A_{4}$

for the 25 percent crack. The results are not uniform as in the baseline case and does not possess the 4-bin pattern as seen for the spall case. The result is not distinctive enough to conclude that damage is present. Figure 24 gives the results for the multiple fault case. The mesh group pattern also suggests that the the transmission is damaged and that the sun gear is the culprit.

\section{SUMMARY AND CONCLUSIONS}

Two methods for detecting sun gear faults have been developed and demonstrated on a full-scale $\mathrm{OH}-58 \mathrm{C}$ transmission. The main goal was to determine if it was possible to isolate the dynamics of the sun gear and detect any damage. This higher level of interrogation improves on any indication of damage by actually confirming sun gear damage as the source, potentially improving the detection rate. 
Sun Gear Spall OSST: 6180@1708 Mast= Full Accelerometer: 1

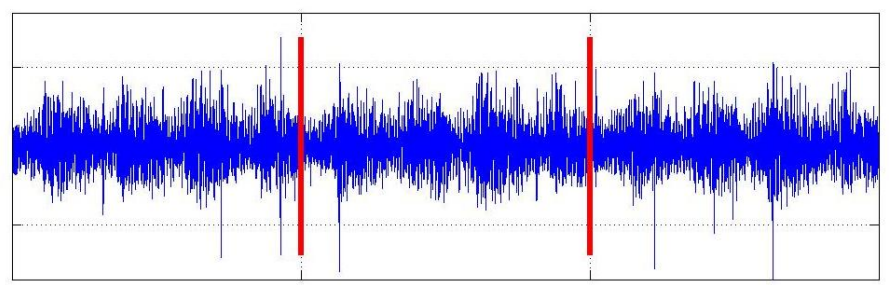

HT1--HT2--HT3

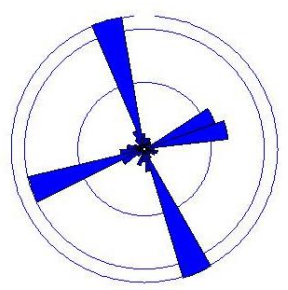

FIGURE 22. GSMM Sun Spall (OSST) $A_{1}$

iear Root Crack 25 Percent Tooth 8 OSST: 6180@1708 Mast= I Accelerometer: 1

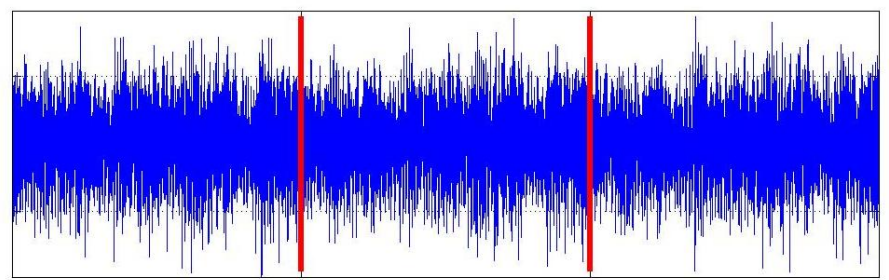

HT1--HT2--HT3

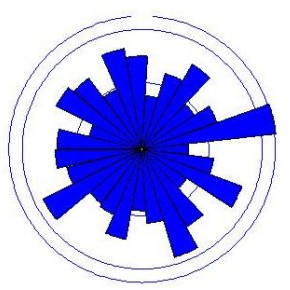

FIGURE 23. GSMM 25 Percent Crack (OSST) $A_{1}$

Planetary gear diagnostics have been demonstrated to be an effective tool to detect planet gear damage, but detecting faults on the sun gear remains a challenge. One reason is because the sun gear's dynamics is determined indirectly, through a meshing planet gear. The dynamics of the planet gear and sun gear are commensurate; thus, time-synchronous averaging could not be used as a separation tool as it may be for the pinion and bevel gear components. In addition, the low hunting tooth ratio between the sun gear and the ring gear allows only a small subset of sun gear teeth to be compared directly. Another obstacle arises
MULT. FAULT SUN UMD: RPM:6294@1717 Accelerometer: 4

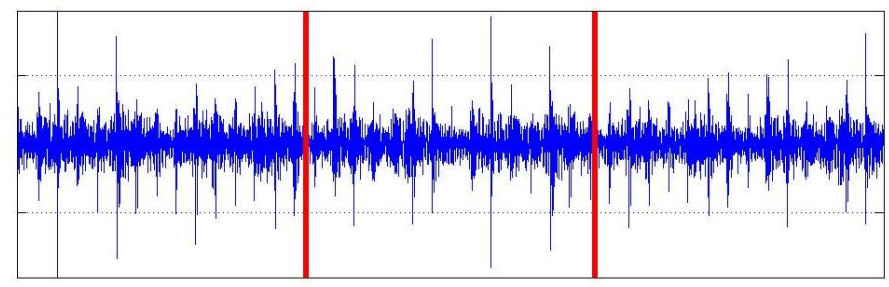

HT1--HT2--HT3

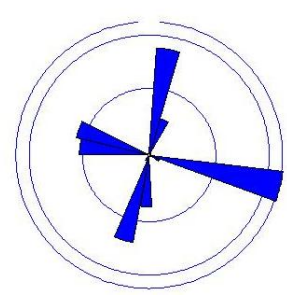

FIGURE 24. GSMM Multiple Faults (UMD) $A_{4}$

from the non-synchronous meshing and unequal spacing of the system's planets. This adds a further level of complexity when working with the waveforms, requiring the need to separate data into different TSA groups.

Both methods performed well in detecting damage for the spall cases. The SGVS -SASP method produced dominant tooth mesh waveforms in its Vibration Separation Waveform with spacings in accord with a sun gear mesh groups. In addition, the details of the individual waveforms are preserved, possibly allowing additional analysis to determine the level of damage for prognosis. Detecting a crack at the $25 \%$ level remains a challenge as no distict waveforms were present in the Vibration Separation Waveform.

The second method exploited the carefully monitored geometry of the planetary system during testing. The geometrically synchronized measurement method matches the signal's response with the sun gear teeth in mesh and tallies a condition indicator parameter. For this study, the sum of the sixth power of the amplitude was used. Consistent with SGVS -SASP, the best responses were obtained for the lower power cases. Plots showed that for the baseline case, there was no biasing of the values of each bin. For the sun gear with a single tooth spall, there was an emergence of mesh groups indicating increased damage. The results for the multiple spall case clearly showed the damage.

\section{ACKNOWLEDGMENT}

The authors would like to thank Dr. Jason Fetty from the U.S. Army Aviation Applied Technology Directorate and Dr. Ryan Ehinger from Bell Helicopter Textron, Inc. for their support and experimental data as part of the Army's OSST pro- 
gram. This research was partially funded by the Government under Agreement No. W911W6-07-2-0003. The views and conclusions contained in this document are those of the authors and should not be interpreted as representing the official policies, either expressed or implied, of the Aviation Applied Technology Directorate or the U.S. Government.

\section{REFERENCES}

[1] DOD, 2007/2012. "Condition based maintenance plus (cbm+) for materiel maintenance". DoDI 4151.22,DoDD 5134.01 .

[2] Stewart, R., July 1997. "Some useful analysis techniques for gearbox diagnostics". Report MHM/R/10/77 - Machine Health Monitoring Group-Institute of Sound and Vibration Research, University of Southampton.

[3] Zakrajsek, J. J., 1994. A review of transmission diagnostics research at NASA lewis research center. Technical Report NASA TM-106746, ARL-TR-599, NASA and the U.S. Army Research Laboratory, December.

[4] Zakrajsek, J., Townsend, D., and Decker, H., 1993. "An analysis of gear fault detection methods as applied to pitting fatigue failure data". NASA TM-105950, AVSCOM TR-92C-035.

[5] Decker, H., Handschuh, R., and Zakrajsek, J., 1994. "An enhancement to the na4 gear vibration diagnostic parameter". NASA TM-106553, ARL-TR-389.

[6] Lewicki, D. G., Decker, H. J., and Shimski, J. T., 1992. Development of a full-scale transmission testing procedure to evaluate advanced lubricants. Technical Report NASA TP3265, AVSCOM TR-91-C-026, NASA and the U.S. Army Aviation Systems Command, August.

[7] Lewicki, D., Spievak, L., Wawrynek, P., Ingraffea, A., and Handschuh, R., July 2000. "Consideration of moving tooth load in gear crack propagation predictions". NASA TM2000-210227.

[8] Hood, A., and Pines, D., 2010. "Sun gear fault detection on an oh-58c helicopter transmission". American Helicopter Society 67th Annual Forum, Virginia Beach, VA.

[9] Choy, F., Huang, S., Zakrajsek, J., Handschuh, R., and Townsend, D., 1994. "Vibration signature analysis of a faulted gear transmission system". NASA TM-106623.

[10] Chin, H., Danai, K., and Lewicki, D., 1992. "Efficient fault diagnosis of helicopter gearbox transmission,". NASA TM106253, TR-92-C-034.

[11] Chin, H., Danai, K., and Lewicki, D., 1992. "Fault detection of helicopter gear boxes using the multi-valued influence matrix method,". NASA TM-106100, AVSCOM TR-92-C-015.

[12] Jammu, V., Danai, K., and Lewicki, D., April 29-May 1, 1997. "Unsupervised connectionist network for fault di- agnosis of helicopter gearboxes". Proceedings of the AHS 53rd Forum, Virginia Beach, VA, pp. 1297-1307.

[13] McFadden, P., April 1986. "Detecting fatigue cracks in gears by amplitude and phase demodulation of the meshing vibration". Journal of Vibration, Acoustics, Stress, and Reliability in Design, 108, pp. 165-170.

[14] Brennan, M., Chen, M., and Reynolds, A., November 1997. "Use of vibration measurements to detect local tooth defects in gears". Sound and Vibration, 31(11), pp. 12-17.

[15] Larder, B., April 29-May 1, 1997. "An analysis of HUMS vibration diagnostic capabilities". Proceedings of the AHS 53rd Forum, Virginia Beach, VA, pp. 1308-1315.

[16] Forrester, B., February 1990. "Analysis of gear vibration in the time-frequency domain". 44th Meeting of the Mechanical Failure Prevention Group.

[17] McFadden, P., and Wang, W., 1991. "Time-frequency domain analysis of vibration signal for machinery diagnostics (ii) the weighted wigner-ville distribution". Report OUEL 1891, University of Oxford.

[18] Samuel, P. D., Pines, D. J., and Lewicki, D. G., 1998. “A comparison of stationary and non-stationary metrics for detecting faults in helicopter gearboxes". Journal of the American Helicopter Society.

[19] Samuel, P. D., and Pines, D. J., 2000. "Classifying helicopter gearbox faults using a normalized energy metric". Smart Materials and Structures, 9.

[20] McFadden, P. D., and Smith, J. D., 1985. "An explanation for the asymmetry of the modulation sidebands about the tooth meshing frequency in epicyclic gear vibration". Proceedings of the Institution of Mechanical Engineers. Part C, Journal of Mechanical Engineering Science, 199(C1), pp. 65-70.

[21] McFadden, P. D., 1991. "A technique for calculating the time domain averages of the vibration of the individual planet gears and sun gear in an epicyclic gearbox". Journal of Sound and Vibration, 144(1), pp. 163-172.

[22] Howard, I. M., 1991. An investigation of vibration signal averaging of individual components in an epicyclic gearbox. Technical Report ARL-PROP-R-185, Australian Department of Defense: Aeronautical Research Laboratory, March.

[23] McFadden, P. D., 1994. "Window functions for the calculation of the time domain averages of the vibration of the individual planet gears and sun gear in an epicyclic gearbox". Journal of Vibration and Acoustics, 116, April, pp. 179187.

[24] Forrester, D., 1998. "A method for the separation of epicyclic planet gear vibration signatures". In Proceedings of Acoustical and Vibratory Surveillance Methods and Diagnostic Techniques, pp. 539-548.

[25] Conroy, J. K., Samuel, P. D., and Pines, D. J., 2003. "Development of a real-time LabVIEW-based testbed for im- 
plementation of planetary gear diagnostic algorithms". In Proceedings of the American Helicopter Society 59th Annual Forum, pp. 1347-1354.

[26] Mark, W., and Hines, J., 2009. "Stationary transducer response to planetary-gear vibration excitation with nonuniform planet loading". Mechanical Systems and Signal Processing, 23(4), p. 1381.

[27] Mark, W., and Hines, J., 2009. "Stationary transducer response to planetary-gear vibration excitation ii". Mechanical Systems and Signal Processing, 23(7), p. 2259.

[28] Bartelmus, W., and Zimroz, R., 2009. "Vibration condition monitoring of planetary gearbox under varying external load". Mechanical Systems and Signal Processing, 23(1), p. 257.

[29] Lewicki, D., LaBerge, K., Ehinger, R., and Fetty, J., 2011. "Planetary gearbox fault detection using vibration separation technique". AHS 67 Annual Forum-Virginia Beach, $V A$.

[30] Mosher, M., 2005. "Results from a new separation algorithm for planetary gear system vibration measurements". ASME 2005 Int. Des. Engineering Technical Conferences and Computer and Information in Engineering Conference, Sept 2005.

[31] Blunt, D. M., Oct. 14, 2004. Synchronous averaging of epicyclic sun gear vibration. US Patent 2004/0200283 Al.

[32] Samuel, P. D., 2003. "Helicopter transmission diagnostics using constrained adaptive lifting. ph.d dissertation". $\mathrm{PhD}$ thesis, University of Maryland, College Park, MD 20742 USA.

[33] Murat Inalpolat, M., 2009. "A theoretical and experimental investigation of modulation sidebands of planetary gear sets ph.d dissertation". PhD thesis, Ohio State University, Ohio.

[34] Krantz, T. L., 1992. "Gear tooth stress measurement of two helicopter planetary stages". NASA TM 105651.

[35] Zakrajsek, J., Handschuh, R., Lewicki, D., and Decker, H., 1995. "Detecting gear tooth fracture in a high contact ratio face gear mesh". NASA/TM-106822, ARL-TR-600.

[36] Mosher, M., and Huff, E., 2004. "Analysis of in-flight measurements from helicopter transmissions". American $\mathrm{He}$ liopter Society 60th Annual Forum, Baltimore, MD 2004.

[37] Mosher, M., Pryor, A., and Huff, E. M., 2002. "Evaluation of standard gear metrics in helicopter flight operation". In 56th Meeting of the Society for Machinery Failure Prevention Technology, Virginia Beach, VA.

[38] Tumer, I., and Huff, E., 2001. "Using triaxial accelerometer data for vibration monitoring of helicopter gearboxes". 2001 ASME Design Engineering Technical Conferences, September.

[39] Lewicki, D., and Ballarini, R., 1997. "Effect of rim thickness on gear crack propagation path". ASME Journal of Mechanical Design, 119(1), pp. 88-95. 